\title{
Transient radiative heat transfer in a suspension of ceria particles undergoing non-stoichiometric reduction
}

\author{
Roman Bader* \\ Research School of Engineering, The Australian National University, Canberra, ACT 0200, Australia \\ Lukas Gampp ${ }^{\dagger}$ \\ Department of Mechanical and Process Engineering, ETH Zurich, Zurich, CH-8092, Switzerland \\ Aldo Steinfeld \\ Department of Mechanical and Process Engineering, ETH Zurich, Zurich, CH-8092, Switzerland \\ and \\ Wojciech Lipiński ${ }^{\S}$ \\ Research School of Engineering, The Australian National University, Canberra, ACT 0200, Australia \\ E-mail:wojciech.lipinski@anu.edu.au
}

\begin{abstract}
Transient radiative heat transfer is analyzed numerically in a directly-irradiated planeparallel medium containing a suspension of ceria particles undergoing non-stoichiometric thermal reduction. The micrometer-sized ceria particles are assumed to be homogenous, non-gray, absorbing, emitting, and anisotropically scattering, while the overall medium is of non-uniform temperature and composition. The unsteady mass and energy conservation equations are solved using the finite-volume method and the explicit Euler time-integration scheme. Radiative transport is modeled using the energy-portioning Monte Carlo raytracing method. The radiative properties are obtained using the Mie theory. The influence of selected model parameters is investigated. In all cases, the time to reach steady-state temperature is shorter than that to reach the equilibrium non-stoichiometry, indicating that the reduction reaction is limited by chemical kinetics, rather than by heat transfer. Increasing particle volume fraction and decreasing particle diameter both increase the optical thickness of the particle suspension, resulting in increasing peak temperature and temperature non-uniformity at steady state. For $5 \mu \mathrm{m}$-dia. particles under 1000-sun irradiation, the peak temperature at steady state ranges from $1860 \mathrm{~K}$ for a particle volume fraction of $f_{\mathrm{v}}=10^{-6}$ to $2200 \mathrm{~K}$ for $f_{\mathrm{v}}=10^{-3}$; the temperature non-uniformity ranges from 20 to $1200 \mathrm{~K}$. For a fixed volume fraction of $f_{\mathrm{v}}=10^{-6}$, decreasing the particle diameter from 20 to 1 $\mu \mathrm{m}$ increases the peak temperature at steady state from 1700 to $2250 \mathrm{~K}$; the temperature non-uniformity increases from 10 to $70 \mathrm{~K}$. Both the final non-stoichiometry and the reaction rate are sensitive to temperature changes. For example, a temperature change of $230 \mathrm{~K}$ from 1860 to $2090 \mathrm{~K}$ nearly doubles the final non-stoichiometry from 0.095 to 0.18 and reduces the time to reach steady state by a factor of 9 from 3.6 to $0.4 \mathrm{~s}$ for the selected set of parameters. The influences of the reaction rate constant and of the morphology-dependent optical properties of the particles on the temperature and non-stoichiometry distributions are discussed.
\end{abstract}

\footnotetext{
* Research Fellow, AIAA non-member.

${ }^{\dagger}$ M.Sc. Student, AIAA non-member.

* Professor, AIAA non-member.

$\S$ Associate Professor, AIAA senior member.
} 


\section{Nomenclature}

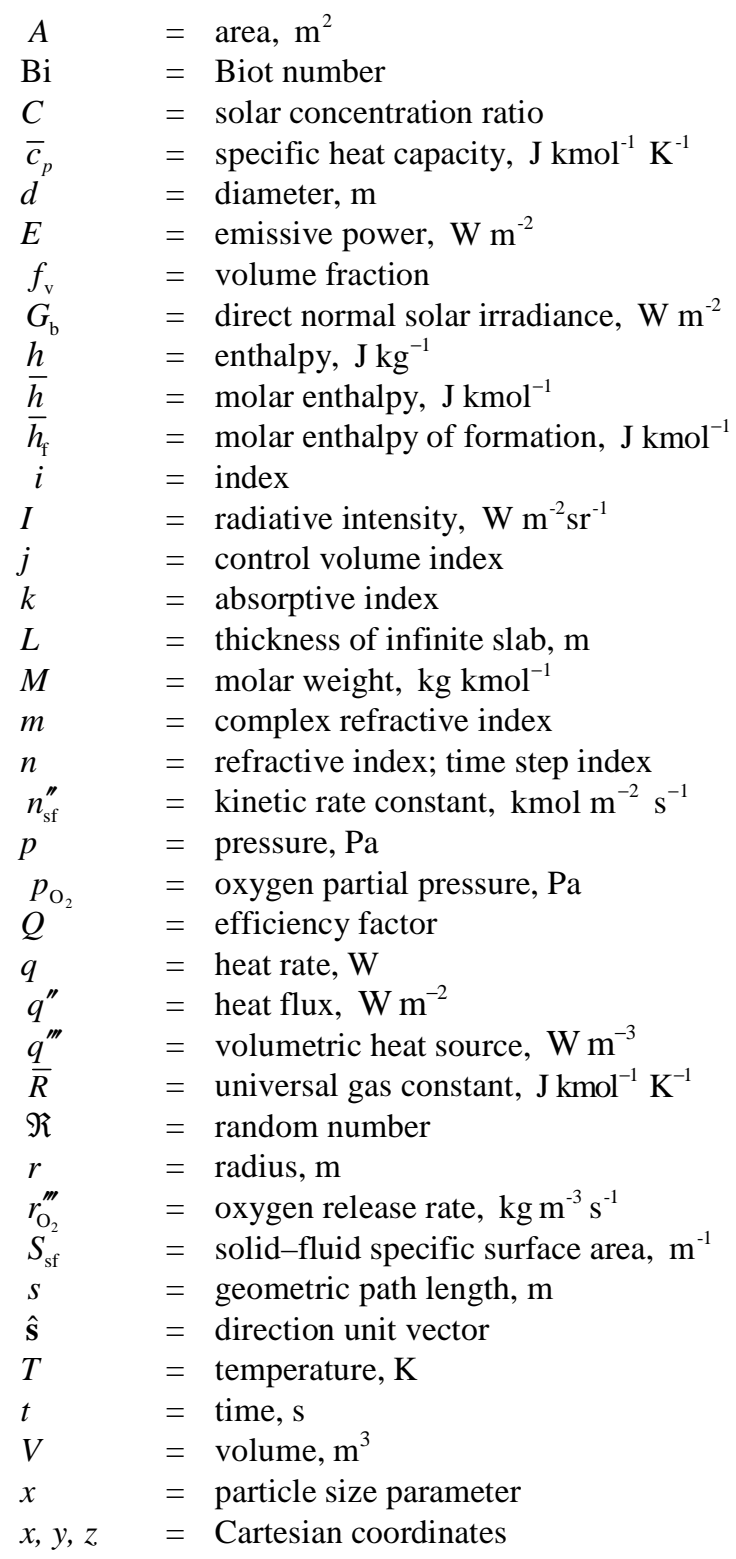

\section{Greek Letters}

$\beta=$ extinction coefficient, $\mathrm{m}^{-1}$

$\Delta \bar{h}_{\mathrm{O}_{2}}^{\mathrm{o}} \quad=$ standard oxidation enthalpy, $\mathrm{J} \mathrm{kmol}^{-1}$

$\Delta \bar{h}_{\mathrm{red}} \quad=$ molar enthalpy of reduction reaction, $\mathrm{J} \mathrm{kmol}^{-1}$

$\Delta \bar{s}_{\mathrm{O}_{2}}^{\mathrm{O}}=$ standard oxidation entropy, $\mathrm{J} \mathrm{kmol}^{-1} \mathrm{~K}^{-1}$

$\Delta t=$ time integration step, $\mathrm{s}$

$\Delta z \quad=$ layer thickness, $\mathrm{m}$

$\Delta \delta \quad=$ change in non-stoichiometry

$\Delta \tau=$ time interval, $\mathrm{s}$

$\delta=$ non-stoichiometry

$\varepsilon=$ emissivity

$\Phi=$ scattering phase function

$\varphi \quad=$ azimuthal angle, $\mathrm{rad}$ 


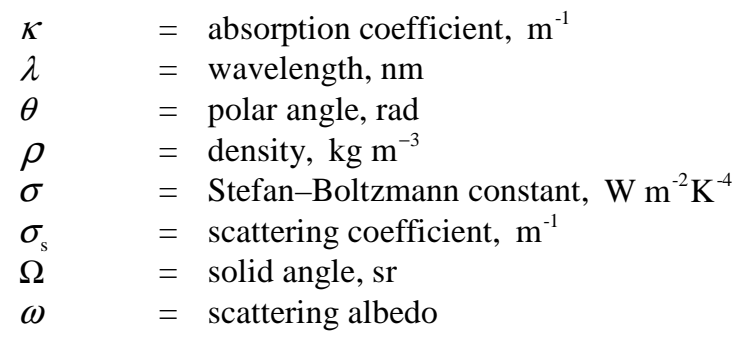

Subscripts

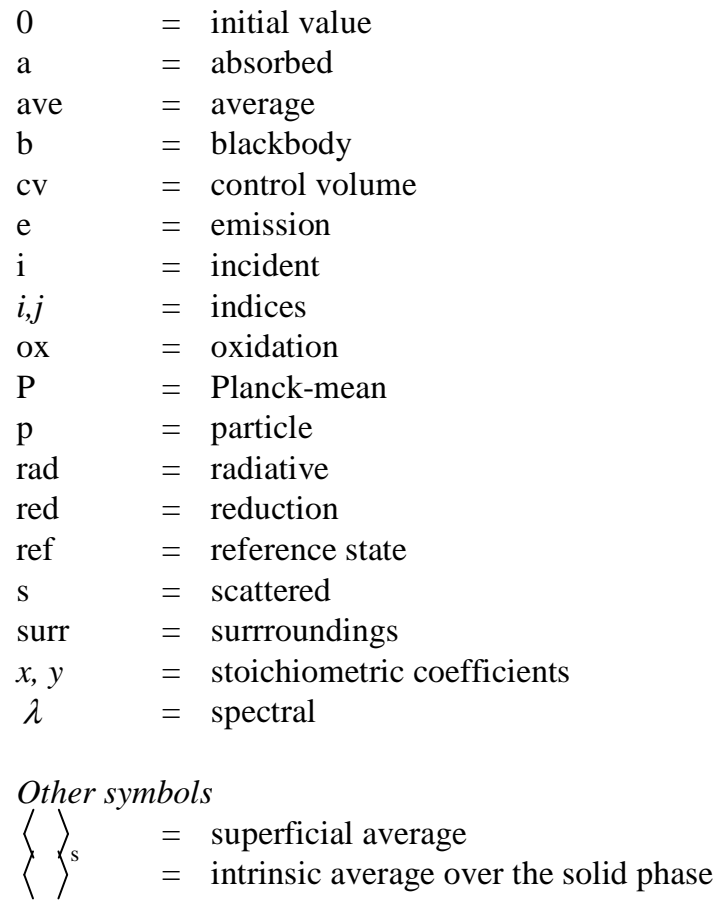

\section{Introduction}

Solar thermochemical splitting of $\mathrm{H}_{2} \mathrm{O}$ and $\mathrm{CO}_{2}$ to produce synthesis gas (syngas), followed by Fischer-Tropsch synthesis, is a promising route to produce $\mathrm{CO}_{2}$-neutral jet fuel. Two-step metal oxide redox cycles have the potential to reach high process efficiencies. ${ }^{1}$ In these processes, a metal oxide is first reduced by exposure to concentrated solar radiation according to:

$$
\frac{1}{\Delta \delta} \mathrm{M}_{x} \mathrm{O}_{y-\delta_{\text {ox }}} \rightarrow \frac{1}{\Delta \delta} \mathrm{M}_{x} \mathrm{O}_{y-\delta_{\text {red }}}+\frac{1}{2} \mathrm{O}_{2}
$$

In reaction (1), $\Delta \delta=\delta_{\text {red }}-\delta_{\text {ox }}>0$ is the change in oxygen non-stoichiometry in the metal oxide, where $\delta_{\text {ox }}$ is the non-stoichiometry in the oxidized state and $\delta_{\text {red }}$ is that in the reduced state. In the second process step, the reducing power of the partially or fully reduced metal oxide is utilized to split $\mathrm{H}_{2} \mathrm{O}$ and $\mathrm{CO}_{2}$ according to:

$$
\begin{gathered}
\frac{1}{\Delta \delta} \mathrm{M}_{x} \mathrm{O}_{y-\delta_{\text {red }}}+\mathrm{H}_{2} \mathrm{O} \rightarrow \frac{1}{\Delta \delta} \mathrm{M}_{x} \mathrm{O}_{y-\delta_{\text {ox }}}+\mathrm{H}_{2} \\
\frac{1}{\Delta \delta} \mathrm{M}_{x} \mathrm{O}_{y-\delta_{\text {red }}}+\mathrm{CO}_{2} \rightarrow \frac{1}{\Delta \delta} \mathrm{M}_{x} \mathrm{O}_{y-\delta_{\text {ox }}}+\mathrm{CO}
\end{gathered}
$$


Cerium dioxide (ceria) is considered as a redox material, due to its potential of reaching oxygen non-stoichiometries of up to $\delta_{\text {red }}=0.25$ without undergoing a phase change, thus maintaining its high oxygen conductivity, and avoiding the need for a separation process to recover the metal oxide from the product gas mixture.,3

In this study, transient radiative heat transfer is analyzed numerically in a directly-irradiated suspension of ceria particles undergoing thermal reduction, Eq. (1). This reactive medium configuration offers high heat transfer rates and high specific surface area, which promotes high reaction rates.

\section{Problem statement}

The model system is shown in Figure 1. It consists of a suspension of micrometer-sized cerium dioxide particles that are fluidized in a flow of nitrogen sweep gas with oxygen content of $10 \mathrm{ppm}$. The particle cloud is directly exposed to concentrated solar radiation. The incident solar radiation is assumed to have the spectral distribution of a blackbody at $5780 \mathrm{~K}$. Due to the high temperatures reached by the particles and the low oxygen content in the sweep gas, the ceria particles undergo partial thermal reduction according to Eq. (1), thereby releasing oxygen into the gas phase. The particle suspension is modeled as an infinite slab containing randomly arranged particles which are surrounded by black plane boundaries at $0 \mathrm{~K}$ (nonparticipating surroundings). The particles are assumed to be homogenous ( $0 \%$ porosity), and their size is assumed constant. They are further treated as isothermal $(\mathrm{Bi}<<1)$, spherical, and independently scattering. The gas phase is assumed to be radiatively non-participating, and heat and mass transfer from/to the gas phase are neglected in the treatment of the gas

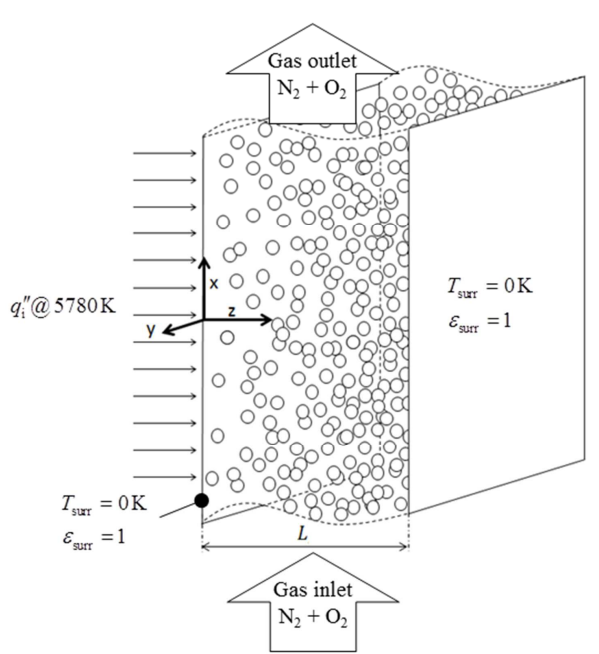

Figure 1. Schematic of the model system. Particle suspension in an infinite slab of width $L$, exposed to concentrated solar radiation. phase. Hence, the sole effects of the gas flow are the fluidization of the particles and the uptake of the oxygen released by the particles. The particle suspension is modeled as uniform, non-gray, absorbing, emitting, anisotropically scattering, and isothermal in a control volume. Two cases are considered for the spatial discretization of the model domain: a multi-layer approximation with free particle flow in $x$ - and $y$-directions and a single layer approximation with particle flow in all directions (perfect mixing). The Monte Carlo ray-tracing method and the Mie theory are used to model the radiative heat transfer in the suspension. The kinetic rate expression for the underlying chemical reaction is adopted from Keene et al. ${ }^{4}$

\section{Governing equations}

In this section, the governing transient volume-averaged mass and energy conservation equations, including the boundary and initial conditions, and the radiative transfer equation are formulated for the suspended of solid ceria particles. The gas phase is assumed to be unaffected by heat transfer in the particle suspension and the oxygen release by the particles. Hence, no mass and energy conservation equations are solved for the fluid phase in this model.

\section{A. Mass conservation}

The volume-averaged mass conservation equation for the solid phase is given by: ${ }^{4}$

$$
\frac{\partial\left\langle\rho_{\mathrm{p}}(t)\right\rangle}{\partial t}=-\left\langle r_{\mathrm{O}_{2}}^{\prime \prime \prime}\right\rangle
$$

where \langle\rangle denotes the superficial volume average and $r_{\mathrm{O}_{2}}^{\prime \prime \prime}$ is the rate of oxygen transfer between solid and gas phase, given in section IV. The volume-averaged density of the solid phase is given by: 


$$
\left\langle\rho_{\mathrm{p}}(t)\right\rangle=\frac{N_{\mathrm{p}} V_{\mathrm{p}}\left\langle\rho_{\mathrm{p}}(t)\right\rangle^{\mathrm{s}}}{V_{\mathrm{cv}}}=\left\langle\rho_{\mathrm{p}}(t)\right\rangle^{\mathrm{s}} f_{\mathrm{v}}
$$

where $\left\langle\rho_{\mathrm{p}}\right\rangle^{\mathrm{s}}$ is the density of the solid phase, averaged over the volume of the solid phase, which can be expressed as a function of the non-stoichiometry $\langle\delta(t)\rangle^{s}$ and the density of stoichiometric ceria, $\rho_{\mathrm{CeO}_{2}}$ :

$$
\left\langle\rho_{\mathrm{p}}\right\rangle^{\mathrm{s}}=\rho_{\mathrm{CeO}_{2}}\left(1-\frac{1}{2} \frac{M_{\mathrm{O}_{2}}\langle\delta(t)\rangle^{\mathrm{s}}}{M_{\mathrm{CeO}_{2}}}\right)
$$

\section{B. Energy conservation}

The volume-averaged energy conservation equation for the solid phase reduces to:

$$
\frac{\partial}{\partial t}\left(\left\langle\rho_{\mathrm{p}}\right\rangle\left\langle h_{\mathrm{p}}\right\rangle^{\mathrm{s}}\right)=\left\langle q_{\mathrm{O}_{2}}^{\prime \prime \prime}\right\rangle-\left\langle\nabla \cdot \mathbf{q}_{\mathrm{rad}}^{\prime \prime}\right\rangle
$$

The enthalpy of non-stoichiometric ceria is calculated from:

$$
h_{\mathrm{p}}=\left(\bar{h}_{\mathrm{CeO}_{2}}-\frac{\delta}{2} \bar{h}_{\mathrm{O}_{2}}+\Delta \bar{h}_{\mathrm{red}}\right) / M_{\mathrm{CeO}_{2-\delta}}
$$

where $\Delta \bar{h}_{\mathrm{red}}$ is the molar enthalpy of the reduction reaction, Eq. (1), $\Delta \bar{h}_{\mathrm{red}}=-1 / 2 \int_{0}^{\delta} \Delta \bar{h}_{\mathrm{O}_{2}}^{\mathrm{o}}\left(\delta^{*}\right) \mathrm{d} \delta^{*}$, and $\bar{h}_{\mathrm{O}_{2}}$ is the molar enthalpy of oxygen. Correlations for $\Delta \bar{h}_{\mathrm{O}_{2}}^{\mathrm{o}}, \bar{h}_{\mathrm{O}_{2}}$, and $\bar{h}_{\mathrm{CeO}_{2}}$ are given in section $\mathrm{V}$. The interphase heat transfer via the oxygen release is given by:

$$
\left\langle q_{\mathrm{O}_{2}}^{\prime \prime \prime}\right\rangle=-\left\langle r_{\mathrm{O}_{2}}^{\prime \prime \prime}\right\rangle h_{\mathrm{O}_{2}}
$$

The radiative source term, $-\left\langle\nabla \cdot \mathbf{q}_{\text {rad }}^{\prime \prime}\right\rangle$, is given in the next section.

\section{Radiative transfer}

The radiative transfer equation takes into account radiation emission, absorption, and anisotropic scattering: ${ }^{5}$

$$
\frac{\mathrm{d} I_{\lambda}}{\mathrm{d} s}=\kappa_{\lambda} I_{\mathrm{b} \lambda}-\kappa_{\lambda} I_{\lambda}-\sigma_{\mathrm{s} \lambda} I_{\lambda}+\frac{\sigma_{\mathrm{s} \lambda}}{4 \pi} \int_{4 \pi} I_{\lambda}\left(\hat{\mathbf{s}}_{\mathrm{i}}\right) \Phi_{\lambda}\left(\hat{\mathbf{s}}_{\mathrm{i}}, \hat{\mathbf{s}}\right) \mathrm{d} \Omega_{i}
$$

Integration over all solid angles and wavelengths yields the radiative source term:

$$
\nabla \cdot \mathbf{q}_{\mathrm{rad}}^{\prime \prime}=\int_{0}^{\infty} \kappa_{\lambda}\left(4 \pi I_{\mathrm{b} \lambda}-\int_{4 \pi} I_{\lambda}(\hat{\mathbf{s}}) \mathrm{d} \Omega\right) \mathrm{d} \lambda
$$

The radiative source term is evaluated numerically as described in section VI.

For the range of values for the size parameter, $x=2 \pi r_{\mathrm{p}} / \lambda$, encountered in the present system, $1 \leq x \leq 10^{2}$, the Mie theory is employed to calculate the scattering and absorption efficiency factors, $Q_{\mathrm{s}}$ and $Q_{\mathrm{a}}$, and the scattering phase function. ${ }^{5,6}$ The efficiency factors are related to the scattering and absorption coefficients via:

$$
\begin{aligned}
& Q_{\mathrm{s} \lambda}=\frac{4}{3} \frac{r_{\mathrm{p}}}{f_{\mathrm{v}}} \sigma_{\mathrm{s} \lambda} \\
& Q_{\mathrm{a} \lambda}=\frac{4}{3} \frac{r_{\mathrm{p}}}{f_{\mathrm{v}}} \kappa_{\lambda}
\end{aligned}
$$

\section{Boundary and initial conditions}

The boundary conditions for the energy equation are:

$$
T_{\text {surr }}=0 \mathrm{~K}, \quad \varepsilon_{\text {surr }}=1, \quad q_{\mathrm{i}}^{\prime \prime}(z=0)=C q_{\text {solar }}^{\prime \prime}
$$


The initial temperature of the particles is set arbitrarily:

$$
\left\langle T_{\mathrm{p}}(t=0,0 \leq z \leq L)\right\rangle=T_{\mathrm{p}, 0}
$$

Initially, the particles are assumed to be in chemical equilibrium. The equilibrium non-stoichiometry of ceria is a function of temperature and oxygen partial pressure: ${ }^{7}$

$$
\Delta \bar{h}_{\mathrm{O}_{2}}^{\mathrm{o}}(\delta)-T \Delta \bar{s}_{\mathrm{O}_{2}}^{\mathrm{o}}(\delta)=\bar{R} T \ln \left(p_{\mathrm{O}_{2}} / p_{\text {ref }}\right)
$$

with $p_{\mathrm{O}_{2}}$, the oxygen partial pressure in the gas phase, equal to the value at the inlet of the model system. Equation (14) with $T=T_{\mathrm{p}, 0}$ yields the initial non-stoichiometry, $\left\langle\delta_{0}\right\rangle$, and Eqs. (5)-(4) yield the initial density, $\left\langle\rho_{\mathrm{p}, 0}\right\rangle$.

\section{Chemical kinetics}

A rate expression for reaction (1) was derived by Keene et al. ${ }^{4}$ In a first step, an expression for the equilibrium constant of the oxygen uptake/release reaction by the ceria was formulated under the assumptions that the oxygen vacancies in the ceria lattice are doubly ionized and that the system behaves like an ideal solution. ${ }^{2}$ Further applying crystal site conservation, electroneutrality, and the law of mass action, an expression was derived for the interphase oxygen mass flux, which is a function of the solid temperature, gas-phase oxygen partial pressure, oxygen nonstoichiometry in the solid, and an unknown kinetic rate constant, $n_{\mathrm{sf}}^{\prime \prime}$ :

$$
\left\langle r_{\mathrm{O}_{2}}^{\prime \prime \prime}\right\rangle=\frac{S_{\mathrm{sf}} N_{\mathrm{p}} M_{\mathrm{O}_{2}}}{V_{\mathrm{cv}}} \frac{n_{\mathrm{sf}}^{\prime \prime}}{2}\left(\langle\delta\rangle^{\mathrm{s}}\right)^{3}\left[\exp \left(\frac{1}{2} \frac{\Delta \bar{h}_{\mathrm{O}_{2}}^{\mathrm{o}}-\left\langle T_{\mathrm{p}}\right\rangle^{\mathrm{s}} \Delta \bar{s}_{\mathrm{O}_{2}}^{\mathrm{o}}}{\bar{R}\left\langle T_{\mathrm{p}}\right\rangle^{\mathrm{s}}}\right)-\left(\frac{p_{\mathrm{O}_{2}}}{p_{\text {ref }}}\right)^{1 / 2}\right]
$$

The kinetic rate constant is treated as a parameter in the present study.

\section{Properties}

\section{A. Optical properties}

Application of the Mie theory requires the knowledge of the complex refractive index of ceria $m_{\lambda}=n_{\lambda}-\mathrm{i} k_{\lambda}$. Several studies have been conducted to determine $m_{\lambda}$ for dense ceria at room temperature, both experimentally and analytically. In the present model, data for $m_{\lambda}$ from three different sources are used and compared: the experimental data obtained by Marabelli \& Wachter ${ }^{8}$ for mono-crystalline ceria, and by Patsalas et al. ${ }^{9}$ for nanocrystalline ceria, and the data obtained by Sun et al. ${ }^{10}$ from first principle calculations for cubic ceria. The values for the refractive index, $n_{\lambda}$, and the absorptive index, $k_{\lambda}$, used in this study are shown in Figure 2 . The complex refractive index is

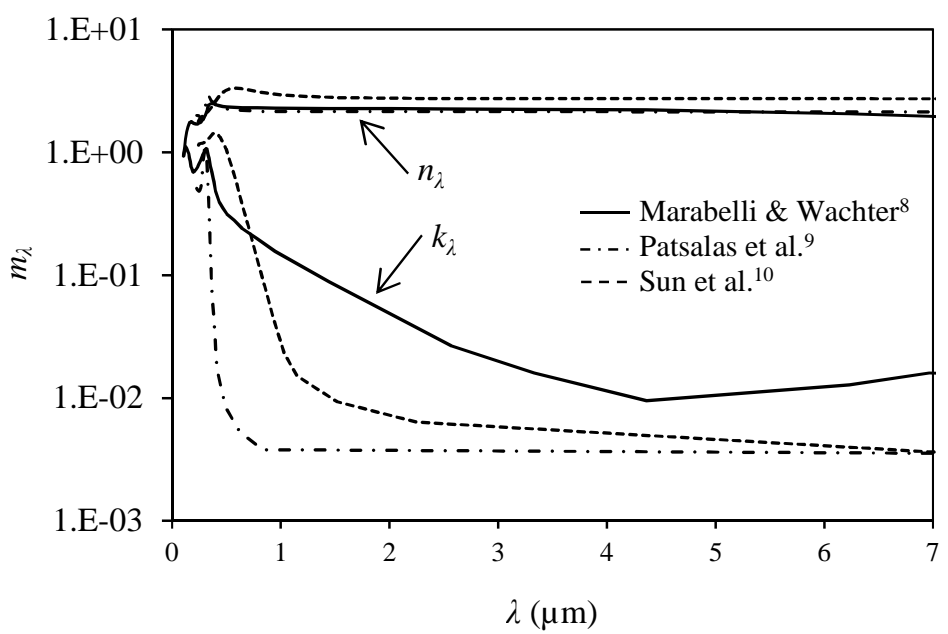

Figure 2. Complex refractive index of ceria. Real $\left(n_{\lambda}\right)$ and imaginary $\left(k_{\lambda}\right)$ parts of the complex refractive index, from Marabelli \& Wachter, ${ }^{8}$ Patsalas et al., ${ }^{9}$ and Sun et al. ${ }^{10}$

6

American Institute of Aeronautics and Astronautics 
assumed to be independent of temperature and non-stoichiometry.

\section{B. Thermophysical properties}

The enthalpy, $\Delta \bar{h}_{\mathrm{O}_{2}}^{\circ}$, and entropy, $\Delta \bar{s}_{\mathrm{O}_{2}}^{\mathrm{o}}$, of reaction (1) have been determined experimentally. ${ }^{7}$ Correlations to describe the data have been obtained by Keene et al.: ${ }^{4}$

$$
\begin{aligned}
& \Delta \bar{h}_{\mathrm{O}_{2}}^{\mathrm{o}}=9.65 \times 10^{7}\left(-10.05+36.01 \delta-417.15 \delta^{2}+4590.21 \delta^{3} \ldots\right. \\
& \left.-31163.99 \delta^{4}+99198.37 \delta^{5}-115948.36 \delta^{6}\right) \\
& \Delta \bar{s}_{\mathrm{O}_{2}}^{\mathrm{o}}=4184.0\left(11.55 \log (\delta)-70.38+224.85 \delta-180.79 \delta^{2}-3999.58 \delta^{3}+9563.11 \delta^{4}\right)
\end{aligned}
$$

The specific heat capacity and enthalpy of ceria and oxygen are calculated with the following correlations, with the parameters listed in Table $1:^{11}$

$$
\begin{gathered}
\bar{c}_{p}=c_{0}+c_{1} T+c_{2} T^{2}+c_{3} T^{-2} \\
\bar{h}(T)=\bar{h}_{\mathrm{f}}+c_{0}\left(T-T_{\text {ref }}\right)+1 / 2 c_{1}\left(T^{2}-T_{\text {ref }}^{2}\right)+1 / 3 c_{2}\left(T^{3}-T_{\text {ref }}^{3}\right)-c_{3}\left(T^{-1}-T_{\text {ref }}^{-1}\right)
\end{gathered}
$$

Table 1. Parameters in Eqs. (17) to calculate the specific heat capacity and enthalpy of ceria and oxygen. ${ }^{11}$

\begin{tabular}{lcc}
\hline Parameter & Ceria & Oxygen \\
\hline$c_{0}$ & $64.81 \times 10^{3}$ & $29.15 \times 10^{3}$ \\
$c_{1}$ & 17.7 & 6.48 \\
$c_{2}$ & 0 & $-1.02 \times 10^{-3}$ \\
$c_{3}$ & $-0.76 \times 10^{9}$ & $-0.18 \times 10^{9}$ \\
$\bar{h}_{\mathrm{f}}\left(\mathrm{J} \mathrm{kmol}^{-1}\right)$ & $-1088.7 \times 10^{6}$ & 0 \\
$T_{\mathrm{ref}}(\mathrm{K})$ & 298 & 298 \\
\hline
\end{tabular}

\section{Numerical solution}

The computational domain is divided into $N_{\mathrm{cv}}$ layers of equal thickness $\Delta z$. The governing equations, Eqs. (3) and (6), are integrated in space using the finite-volume technique and over time with the explicit Euler time integration scheme. The radiative source term, $-\left\langle\nabla \cdot \mathbf{q}_{\text {rad }}^{\prime \prime}\right\rangle$, is determined in every time step using the energyportioning Monte Carlo ray tracing method. ${ }^{12}$ This involves the emission of a prescribed number of ray bundles from within the computational domain as well as from its boundaries, and following their paths through the participating medium until their power is reduced by absorption below a threshold level or they are lost through the medium boundaries.

\section{A. Integrated mass and energy conservation equations}

The discrete, integrated mass and energy conservation equations are:

$$
\begin{gathered}
\left\langle\rho_{\mathrm{p}}\right\rangle_{j}^{n+1}=\left\langle\rho_{\mathrm{p}}\right\rangle_{j}^{n}-\left\langle r_{\mathrm{O}_{2}}^{\prime \prime \prime}\right\rangle \Delta t \\
\left(\left\langle T_{\mathrm{p}}\right\rangle_{j}^{\mathrm{s}}\right)^{n+1}=\left(\left\langle T_{\mathrm{p}}\right\rangle_{j}^{\mathrm{s}}\right)^{n}+\frac{\Delta t}{\left\langle\rho_{\mathrm{p}}\right\rangle_{j}^{n} c_{p, \mathrm{p}, j}}\left(-\frac{1}{A_{\mathrm{cv}} \Delta z_{V_{\mathrm{cv}, j}}} \int\left\langle\nabla \cdot \mathbf{q}_{\mathrm{rad}}^{\prime \prime \prime}\right\rangle \mathrm{d} V+\left\langle r_{\mathrm{O}_{2}}^{\prime \prime \prime}\right\rangle_{j}\left(\left\langle h_{\mathrm{p}}\right\rangle_{j}^{\mathrm{s}}-h_{\mathrm{O}_{2}, j}\right)\right)^{n}
\end{gathered}
$$

\section{B. Radiation emission inside the medium}

Prescribing $N_{\text {ray,e }}$, the total number of ray bundles traced in each time step to represent the radiative power emitted within the medium, the radiative power carried by a ray bundle at the point of emission is: 


$$
q_{\text {ray,e }}=\frac{1}{N_{\text {ray,e }}} \int_{V} 4 \kappa_{\mathrm{P}} \sigma T^{4} \mathrm{~d} V
$$

The model domain is discretized into $N_{\mathrm{cv}}\left(j=1, \ldots, N_{\mathrm{cv}}\right)$ isothermal layers of unit cross-section and thickness $\Delta z$, $V_{\mathrm{cv}}=A_{\mathrm{cv}} \Delta z$, where $A_{\mathrm{cv}}=1 \mathrm{~m}^{2}$, with uniform properties in each layer. The discrete form of Eq. (20) is:

$$
q_{\mathrm{ray}, \mathrm{e}}=\frac{1}{N_{\mathrm{ray}, \mathrm{e}}} \sum_{j=1}^{N_{\mathrm{cv}}} 4 \kappa_{\mathrm{P}, j} \sigma\left(\left\langle T_{\mathrm{p}, j}\right\rangle^{\mathrm{s}}\right)^{4} V_{\mathrm{cv}}=\frac{A_{\mathrm{cv}} \Delta z}{N_{\text {ray }, e}} \sum_{j=1}^{N_{\mathrm{cv}}} 4 \kappa_{\mathrm{P}, j} \sigma\left(\left\langle T_{\mathrm{p}, j}\right\rangle^{\mathrm{s}}\right)^{4}
$$

The number of rays emitted from within layer $j$ is found from:

$$
N_{\text {ray,e, }, j}=\operatorname{nint}\left(\frac{1}{q_{\text {ray,e }}} \int_{V_{\mathrm{er}, j}} 4 \kappa_{\mathrm{p}} \sigma T_{\mathrm{p}}^{4} \mathrm{~d} V\right)=\operatorname{nint}\left(\frac{1}{q_{\mathrm{ray}, \mathrm{e}}} 4 \kappa_{\mathrm{p}, j} \sigma\left(\left\langle T_{\mathrm{p}, j}\right\rangle^{\mathrm{s}}\right)^{4} A_{\mathrm{cv}} \Delta z\right)
$$

with $q_{\text {ray, }}$ obtained from Eq. (21). The location of emission of a ray bundle within layer $j$ is determined from:

$$
z_{\mathrm{e}, j}=(j-1+\Re) \Delta z
$$

where $\Re$ denotes a random number from the uniform range $(0,1)$. The direction of emission in polar coordinates is found from:

$$
\begin{gathered}
\varphi=2 \pi \Re \\
\theta=\cos ^{-1}(1-2 \Re)
\end{gathered}
$$

The wavelength $\lambda$ of an emitted ray is determined from:

$$
\Re=\frac{1}{\kappa_{\mathrm{p}, j} \sigma\left(\left\langle T_{\mathrm{p}, j}\right\rangle^{\mathrm{s}}\right)^{4}} \int_{0}^{\lambda} \kappa_{\lambda}\left(\lambda^{*},\left\langle T_{\mathrm{p}, j}\right\rangle^{\mathrm{s}}, p\right) E_{\lambda \mathrm{b}}\left(\lambda^{*},\left\langle T_{\mathrm{p}, j}\right\rangle^{\mathrm{s}}\right) \mathrm{d} \lambda^{*}
$$

\section{Externally incident radiation}

Concentrated solar radiation is assumed to be incident at $z=0$ with direction parallel to the $z$-axis. Prescribing the solar concentration ratio $C$ and the number $N_{\text {ray,i }}$ of rays traced in each time step to simulate the incident solar radiation, the radiative power carried by a ray bundle at $z=0$ is:

$$
q_{\mathrm{ray}, \mathrm{i}}=\frac{C G_{\mathrm{b}} A_{\mathrm{cv}}}{N_{\mathrm{ray}, \mathrm{i}}}
$$

\section{Ray tracing}

The ray's travel distance within the medium, $s$, between two attenuation points is obtained by numerically solving:

$$
\Re=\frac{I_{\lambda}(s)}{I_{\lambda}(0)}=\exp \left(-\int_{0}^{s} \beta_{\lambda}\left(s^{*}\right) \mathrm{d} s^{*}\right)
$$

At the location of attenuation, the energy carried by the ray, $q_{\text {ray }}$, is partitioned into absorbed and scattered fractions according to:

$$
\begin{gathered}
q_{\text {ray,a }}=\left(1-\omega_{\lambda}\right) q_{\text {ray }} \\
q_{\text {ray }, \mathrm{s}}=\omega_{\lambda} q_{\text {ray }}
\end{gathered}
$$

\footnotetext{
* With an ideal solar concentrator with a concentration ratio of $C=1000$, the concentrated solar radiation would be incident within a cone half-angle of $8.5^{\circ}$.
}

8

American Institute of Aeronautics and Astronautics 
where $\omega_{\lambda}$ is the scattering albedo. The new travel direction of the scattered fraction of the ray is determined relative to the ray travel direction before the attenuation event, using the scattering phase function and random numbers:

$$
\begin{gathered}
\Re=\frac{1}{2} \int_{0}^{\theta} \Phi_{\lambda}\left(\theta^{*}\right) \sin \theta^{*} \mathrm{~d} \theta^{*} \\
\varphi=2 \pi \Re
\end{gathered}
$$

The ray tracing of a ray bundle is terminated when the scattered fraction of the ray energy is below $0.01 \%$ of the ray's initial energy. Once all $N_{\text {ray,e }}+N_{\text {ray,i }}$ rays have been traced through the model domain, the integrated radiative source term is calculated from:

$$
-\int_{V_{\mathrm{cv}, j}}\left\langle\nabla \cdot \mathbf{q}_{\mathrm{rad}}^{\prime \prime}\right\rangle \mathrm{d} V=\sum_{i=1}^{N_{\mathrm{a}, j}} q_{\mathrm{ray}, \mathrm{a}, j, i}-4 \kappa_{\mathrm{P}, j} \sigma\left(\left\langle T_{\mathrm{p}, j}\right\rangle^{\mathrm{s}}\right)^{4} A_{\mathrm{cv}} \Delta z
$$

The first term corresponds to the radiative energy absorbed in layer $j$, where $N_{\mathrm{a}, j}$ is the total number of attenuation events in layer $j$ (of both, internally emitted and externally incident rays), and $q_{\text {ray,a, }, i, i}$ is the absorbed energy at each attenuation event. The second term corresponds to the radiative energy emitted from within the layer.

\section{Results}

A parametric study has been conducted to analyse the influence of different model parameters on the transient and steady-state temperature and non-stoichiometry distributions in the particle suspension. Table 2 gives an overview of the selected baseline values for the model parameters, and the ranges over which selected parameters were varied. Parameters not varied were set to the baseline value, unless stated otherwise. The time integration step required an adjustment depending on the volume fraction of the particle suspension; its value was set between $5 \times 10^{-3}$ and $1 \times 10^{-2} \mathrm{~s}$. The total number of rays traced in each time step, $N_{\text {ray }}=N_{\text {ray,e }}+N_{\text {ray, },}$, was selected in the range of $10^{6}-2 \times 10^{7}$.

The evolution of the temperature profile in the particle suspension is shown in Figure 3 for different particle volume fractions. Low volume fractions correspond to low thermal capacity of the suspension and low optical thickness, resulting in short heat-up time and uniform temperature distribution. The time to reach the steady-state temperature distribution is indicated above the black curve, which is the asymptotic temperature profile. The steadystate energy balances for the two cases with $f_{\mathrm{v}}=10^{-6}$ and $f_{\mathrm{v}}=10^{-4}$ are compared in Table 3 . With $f_{\mathrm{v}}=10^{-6}$, the suspension absorbs only $1.4 \%$ of the incident solar radiation, the rest being transmitted or reflected (the scattering and absorption coefficients are proportional to the volume fraction, Eqs. (11)). In contrast, for $f_{\mathrm{v}}=10^{-4} 75 \%$ of the incident solar radiation is absorbed and re-radiated to the environment by the particle suspension, leading to a higher

Table 2. Model parameters.

\begin{tabular}{lccc}
\hline Parameter & Baseline value & \multicolumn{2}{c}{ Range } \\
\hline Layer thickness, $L(\mathrm{~m})$ & 0.1 & & \\
Solar concentration ratio, $C$ & 1000 & & \\
Direct normal irradiance, $G_{\mathrm{b}}\left(\mathrm{W} \mathrm{m}^{-2}\right)$ & 1000 & & \\
Initial particle temperature, $T_{\mathrm{v} .0}(\mathrm{~K})$ & 300 & & \\
Oxygen partial pressure, $p_{\mathrm{O},}(\mathrm{atm})$ & $10^{-5}$ & & \\
Total pressure, $p(\mathrm{~atm})$ & 1 & $10^{-6}$ & $10^{-3}$ \\
Volume fraction, $f_{\mathrm{v}}(\mu \mathrm{m})$ & $10^{-6}$ & 1 & 20 \\
Particle diameter, $d_{\mathrm{v}}\left(\mu{ }^{\prime \prime}\left(\mathrm{mol} \mathrm{m}^{-2} \mathrm{~s}^{-1}\right)\right.$ & 5 & 0.1 & 10 \\
Kinetic rate constant, $n_{\mathrm{O},}$ & 1 & 1 & 50 \\
Number of layers, $N_{\mathrm{cv}}$ & 50 & Marabelli $^{8}$ & Sun et al. ${ }^{10}$ \\
Complex refractive index, $m_{\lambda}$ & Patsalas et al. $^{9}$ & \& Wachter & \\
\hline
\end{tabular}


(a)

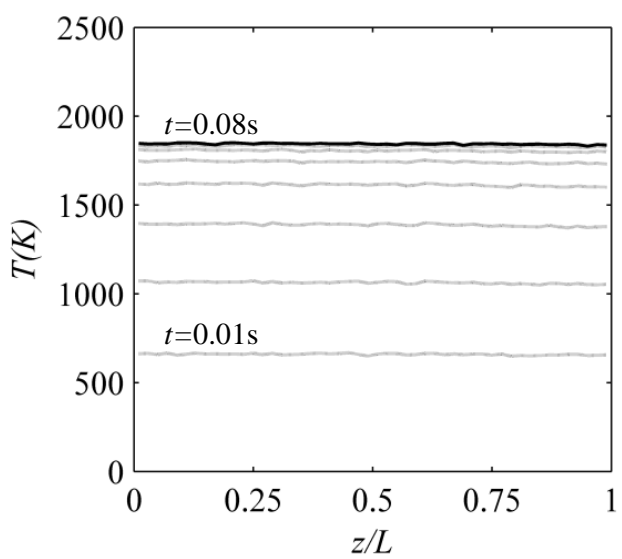

(c)

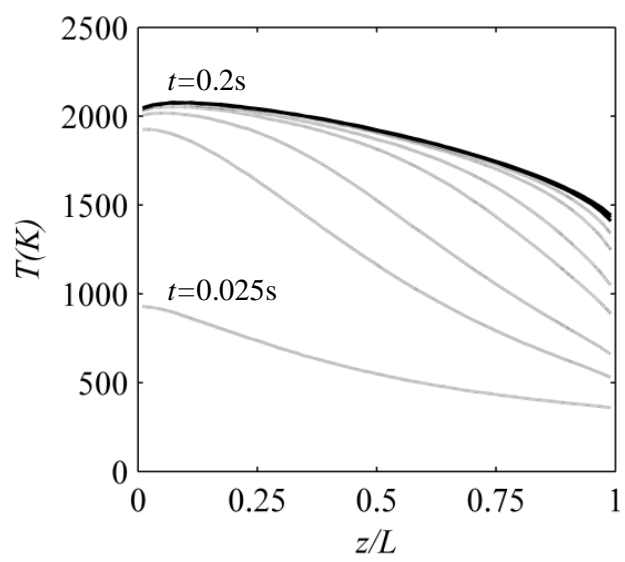

(b)

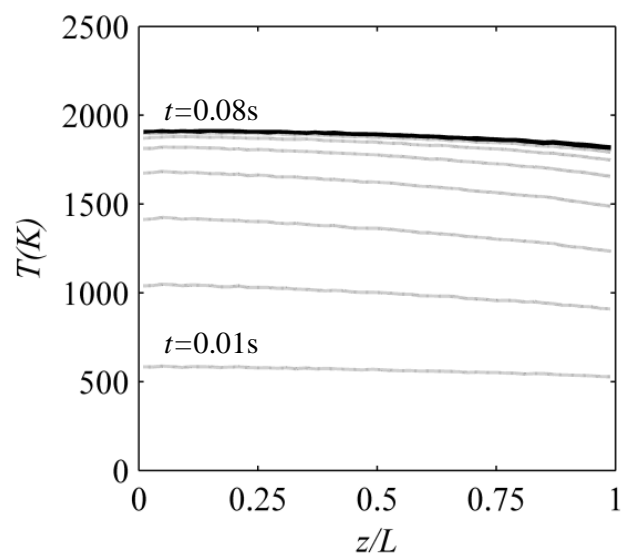

(d)

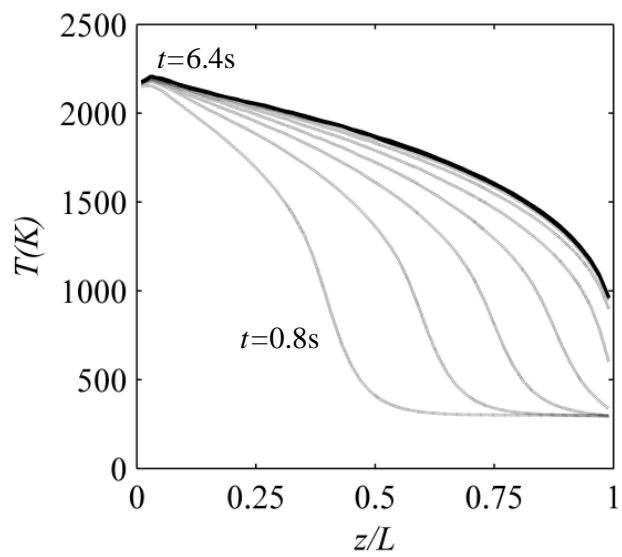

Figure 3. Evolution of the temperature profile in the particle suspension for different volume fractions. (a) $f_{\mathrm{v}}=10^{-6}$, (b) $f_{\mathrm{v}}=10^{-5}$, (c) $f_{\mathrm{v}}=10^{-4}$, and (d) $f_{\mathrm{v}}=10^{-3}$. The gray curves show the transient temperature profiles at different time intervals $\Delta \tau:(a)-(b): \Delta \tau=0.01 \mathrm{~s},(c): \Delta \tau=0.025 \mathrm{~s},(d): \Delta \tau=0.8 \mathrm{~s}$. The black curves are the steady-state temperature profiles.

Table 3. Comparison of the steady-state energy balances for the cases with volume fractions of $f_{\mathrm{v}}=10^{-6}$ and $f_{v}=10^{-4}$ (in \% of the incident solar power).

\begin{tabular}{lccc}
\hline Volume fraction & Transmitted & $\begin{array}{c}\text { Absorbed and re-radiated } \\
\text { (left / right) }\end{array}$ & Reflected \\
\hline$f_{\mathrm{v}}=10^{-6}$ & 98 & $1.4(0.7 / 0.7)$ & 0.6 \\
$f_{\mathrm{v}}=10^{-4}$ & 9.8 & $75.1(46.8 / 28.3)$ & 15.1 \\
\hline
\end{tabular}

peak temperature at equilibrium (Figure 3). For the cases with significant temperature non-uniformities (Figures $3 \mathrm{c}$ and d), the peak equilibrium temperature occurs at a short distance from the irradiated boundary, where the incident solar radiative flux is high and the particles are surrounded by other hot particles.

The evolution of the non-stoichiometry distribution in the particle suspension is shown Figures 4a-c for particle volume fractions of $f_{\mathrm{v}}=10^{-4}, 10^{-5}$, and $10^{-6}$. In all three cases, the non-stoichiometry distribution takes longer to reach steady state than the temperature distribution, suggesting that the process is limited by chemical kinetics rather than by the heating rate, for the selected parameters. The equilibrium non-stoichiometry strongly depends on the temperature, $T_{\mathrm{p}}$, (Eq. 14). This results in strong non-uniformities in the non-stoichiometry distribution within the 
(a)

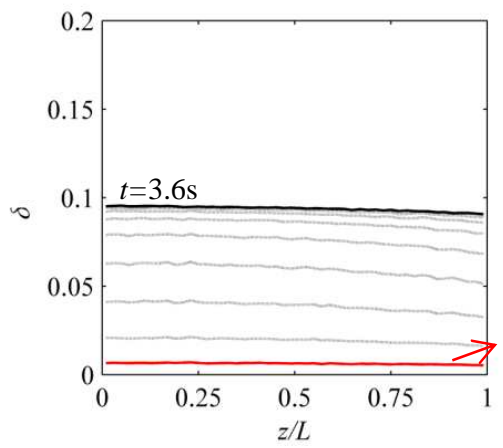

(b)

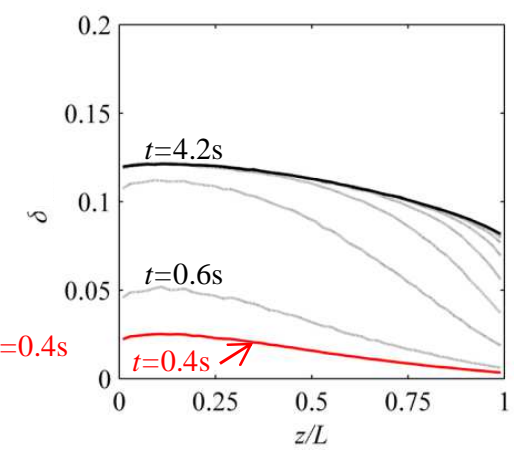

(c)

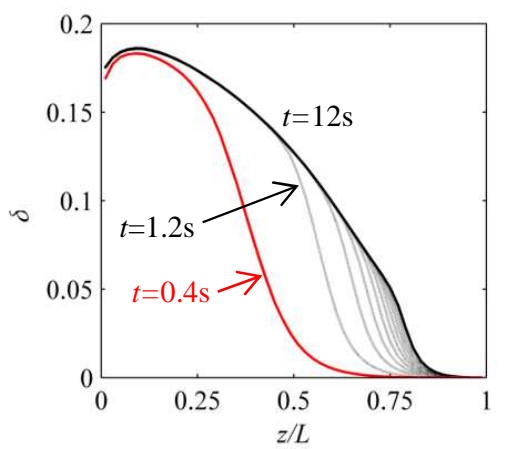

Figure 4. Evolution of the non-stoichiometry distribution in the particle suspension for different volume fractions. (a) $f_{\mathrm{v}}=10^{-6},(b) f_{\mathrm{v}}=10^{-5}$, and (c) $f_{\mathrm{v}}=10^{-4}$. The initial non-stoichiometry is $\left\langle\delta_{0}\right\rangle \approx 0$. The gray curves show the transient non-stoichiometry distributions at different time intervals $\Delta \tau:(a): \Delta \tau=0.4 s,(b): \Delta \tau=0.6 s,(c)$ : $\Delta \tau=1.2 \mathrm{~s}$. The black curves are the final non-stoichiometry distributions. The red curves are the non-stoichiometry distributions after $0.4 \mathrm{~s}$.

(a)

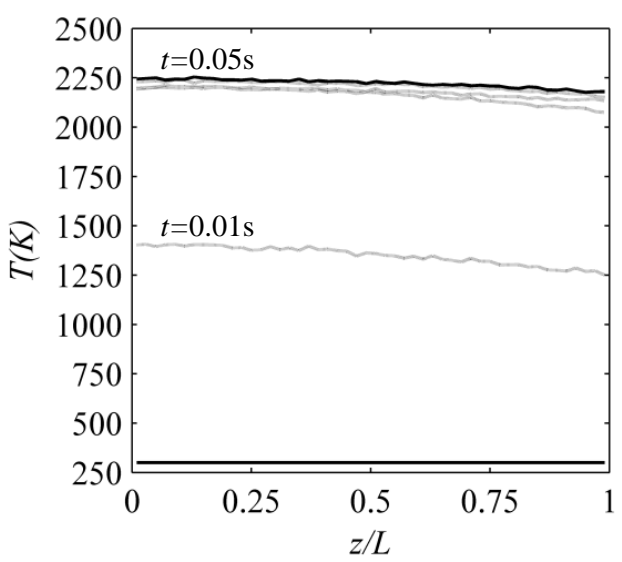

(c)

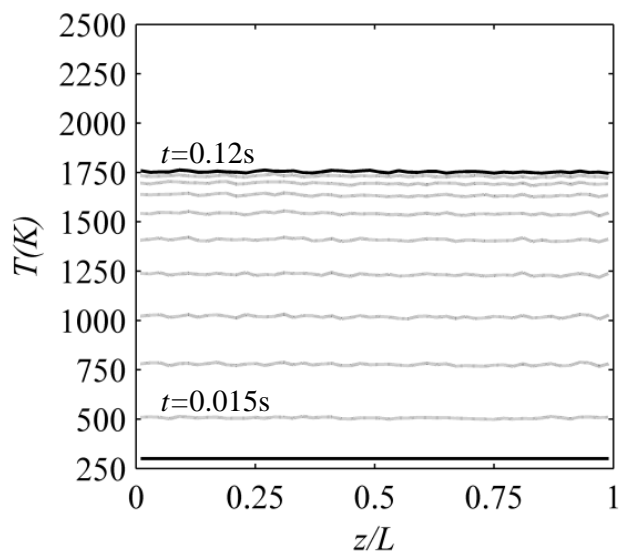

(b)

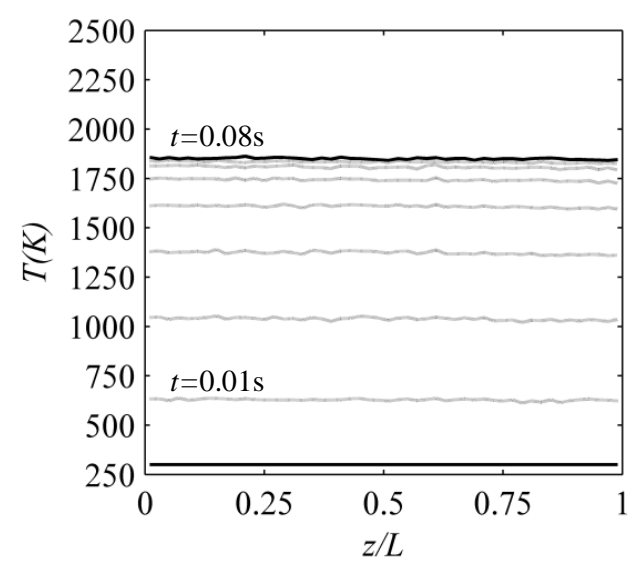

(d)

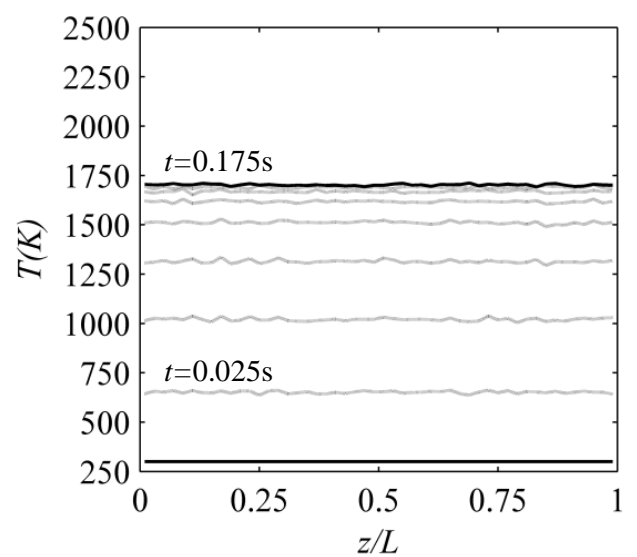

Figure 5. Evolution of the temperature profile in the particle suspension for different particle diameters. $(a)$ $d_{\mathrm{p}}=1 \mu \mathrm{m},(b) d_{\mathrm{p}}=5 \mu \mathrm{m},(c) d_{\mathrm{p}}=10 \mu \mathrm{m}$, and $(d) d_{\mathrm{p}}=20 \mu \mathrm{m}$. The gray curves show the transient temperature profiles at different time intervals $\Delta \tau:(a)-(c): \Delta \tau=0.01 \mathrm{~s},(d): \Delta \tau=0.025 \mathrm{~s}$. The black curves are the steady-state temperature profiles. 
particle suspension even for relatively uniform temperature distributions such as for $f_{\mathrm{v}}=10^{-5}$. In regions where the temperature remains below approx. $1700 \mathrm{~K}$, particles do not react to a significant extent (Figure 4c).

The reaction rate, Eq. (15), also strongly depends on the temperature $T_{\mathrm{p}}$. This is illustrated by the red curves in Figure 4 which show the non-stoichiometry distribution after $0.4 \mathrm{~s}$ of simulation time. Within this time period, the temperature distribution reaches steady state in all three cases (Figure 3a-c). However, the peak steady-state temperatures differ between the three cases $\left(f_{\mathrm{v}}=10^{-6}\right.$ : $\left.1860 \mathrm{~K}, f_{\mathrm{v}}=10^{-5}: 1920 \mathrm{~K}, f_{\mathrm{v}}=10^{-4}: 2090 \mathrm{~K}\right)$. These differences in temperature lead to strong differences in the reaction rates, and consequently in the peak nonstoichiometries reached after $0.4 \mathrm{~s}$. The effect of the temperature dependence of the reaction rate can also be well observed from the red curve in Figure $4 \mathrm{c}$ alone: near $z / L=0$, where the temperature is highest, the steady-state non-stoichioimetry has nearly been reached after $0.4 \mathrm{~s}$ of simulation time, whereas the non-stoichiometry is still far away from the steady-state value in the cooler regions.

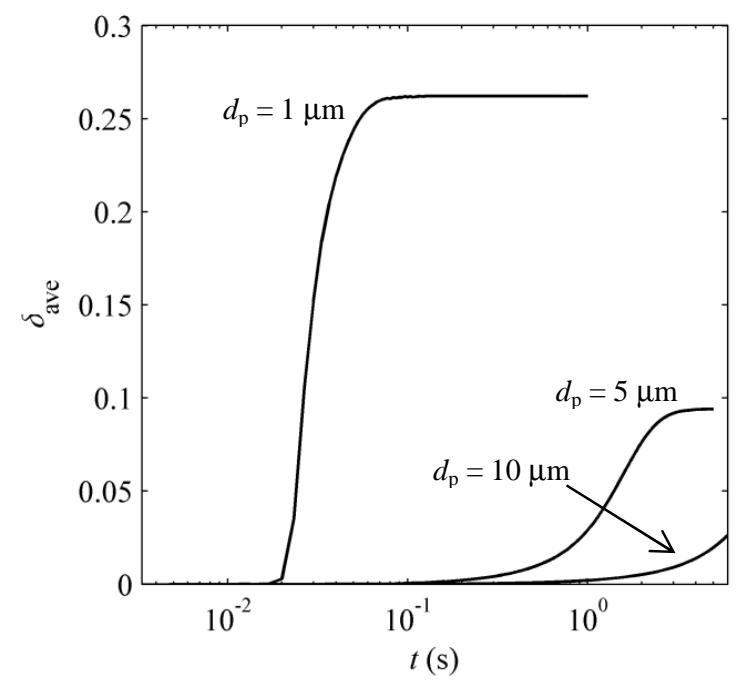

Figure 6. Evolution of average non-stoichiometry in the particle suspension for different particle diameters.

Despite the large differences in the non-stoichiometry distributions, the average equilibrium non-stoichiometry in the particle suspension varies by less than $20 \%$ among the three cases shown in Figure 4.

Figure 5 shows the evolution of the temperature profile in the particle suspension for different particle diameters. Decreasing particle diameter leads to increasing absorption and scattering coefficients, particularly over the spectrum relevant for solar radiation. This leads to increasing heating rate, higher steady-state temperature, and faster reaction rate with decreasing particle diameter. The latter can be seen in Figure 6, which shows the evolution of the average non-stoichiometry in the particle suspension for different particle diameters. Despite the higher radiation attenuation, the temperature distribution is relatively uniform throughout the particle suspension for $d_{\mathrm{p}}=1 \mu \mathrm{m}$.

The influence of the kinetic rate constant, $n_{\mathrm{O}_{2}}^{\prime \prime}$, on the evolution of the non-stoichiometry is shown in Figure 7, and compared to the evolution of the temperature profile. The rate constant strongly influences the evolution of the non-stoichiometry. The time to reach steady state increases from $0.38 \mathrm{~s}$ for $n_{\mathrm{O}_{2}}^{\prime \prime}=10 \mathrm{kmol} \mathrm{m}^{-2} \mathrm{~s}^{-1}$ to $40 \mathrm{~s}$ (not shown

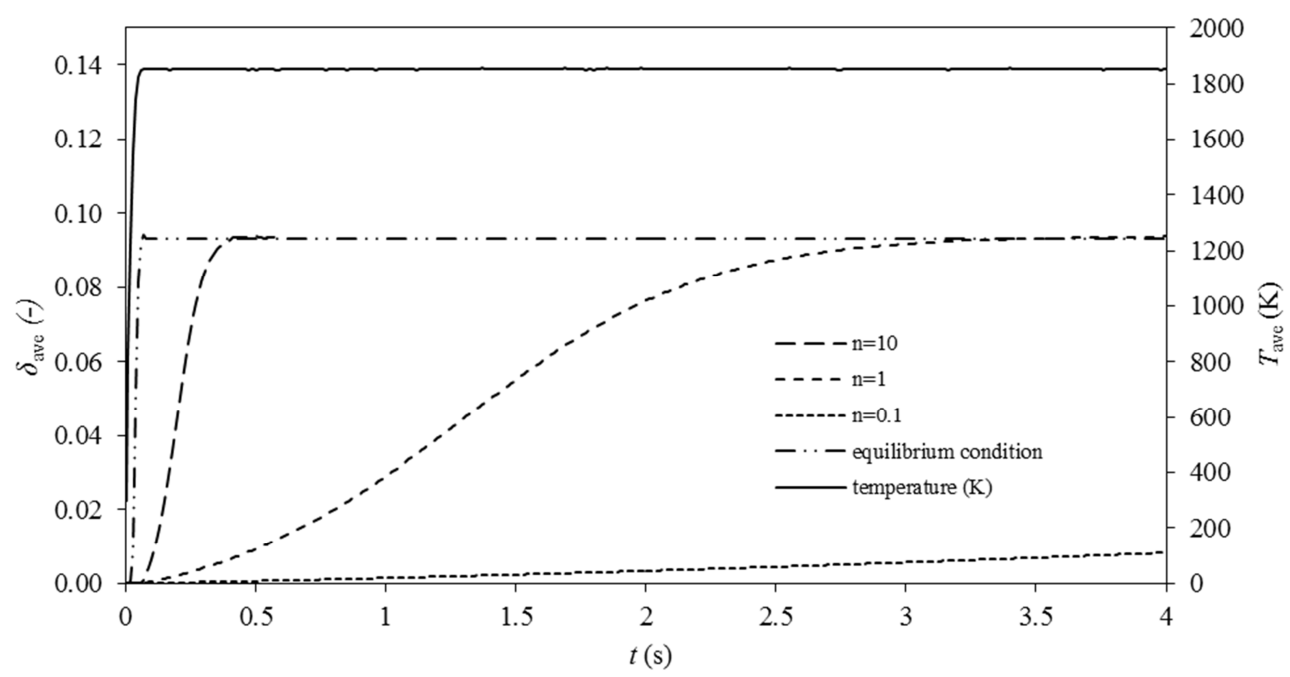

Figure 7. Evolution of average non-stoichiometry (left $y$-axis), and temperature (right $y$-axis) in the particle suspension for different kinetic rate constants, $n_{\mathrm{sf}}^{\prime \prime}$, in $\mathrm{kmol} \mathrm{m}^{-2} \mathrm{~s}^{-1}$. 
(a)

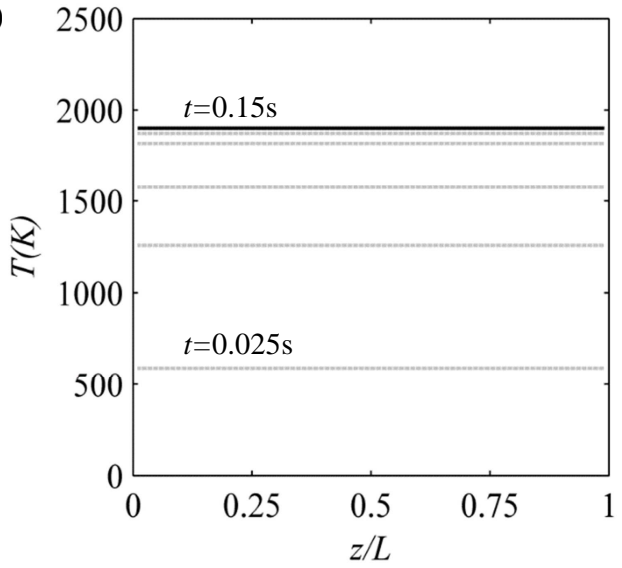

(b)

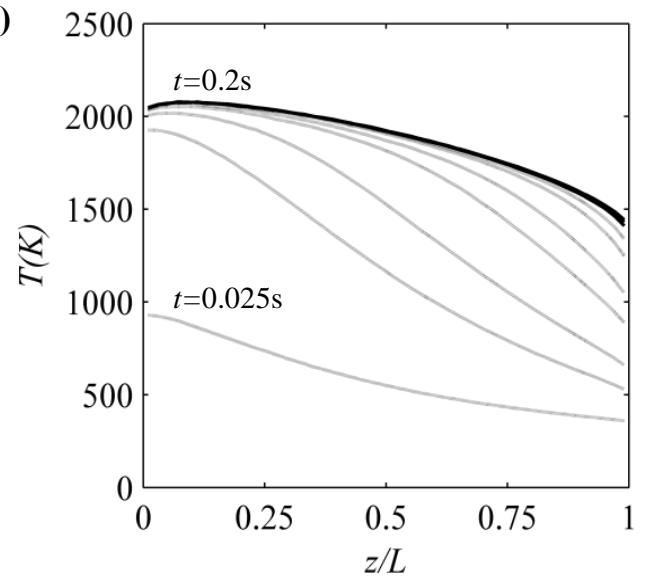

Figure 8. Evolution of the temperature profile in the particle suspension for different mixing assumptions. (a) $N_{\mathrm{cv}}=1,(b) N_{\mathrm{cv}}=50$. The parameters are the baseline values, except for $f_{\mathrm{v}}=10^{-4}$. The gray curves show the transient temperature profiles at the time interval $\Delta \tau=0.025 \mathrm{~s}$. The black curves are the steady-state temperature profiles.

in the graph) for $n_{\mathrm{O}_{2}}^{\prime \prime}=0.1 \mathrm{kmol} \mathrm{m}^{-2} \mathrm{~s}^{-1}$. For $n_{\mathrm{O}_{2}}^{\prime \prime} \rightarrow \infty$, the reaction rate is heat transfer-limited, and the nonstoichiometry corresponds to the equilibrium non-stoichiometry at any time $t$, as shown by the dash-dotted line.

The baseline case assumes that every particle is confined to one of the $N_{\mathrm{cv}}$ parallel layers. Such a situation can be representative of falling particles whose trajectories are relatively undisturbed by the gas flow. On the other hand, if the particles are intensely mixed by the gas flow, the particle suspension can be assumed to be perfectly mixed. In this case, $N_{\mathrm{cv}}=1$. Figure 8 compares the transient temperature distribution in the particle suspension for the cases with $N_{\mathrm{cv}}=1$ and $N_{\mathrm{cv}}=50$, and volume fraction $f_{\mathrm{v}}=10^{-4}$. Ideal mixing of the particles $\left(N_{\mathrm{cv}}=1\right)$ leads to perfectly uniform temperature and hence non-stoichiometry distributions in the particle suspension.

Figure 9 shows the spectral scattering and absorption coefficients obtained using the three data sets for the complex refractive index by Marabelli \& Wachter, ${ }^{8}$ Patsalas et al., ${ }^{9}$ and Sun et al. ${ }^{10}$ While the values for the scattering coefficient are comparable for the three models, the absorption coefficient shows significant differences between the models in the visible and infrared wavelength ranges. The evolution of the temperature profile in the particle suspension for the three cases is shown in Figure 10. Higher absorption coefficient in the visible (0.4-0.7 $\mu \mathrm{m})$ and near-infrared $(0.7-1.4 \mu \mathrm{m})$ wavelength ranges leads to stronger absorption of solar radiation by the particle suspension resulting in higher heating rates. On the other hand, higher absorption coefficient in the near- and midinfrared regions leads to higher radiation emission by the particle suspension resulting in lower equilibrium temperature.

(a)

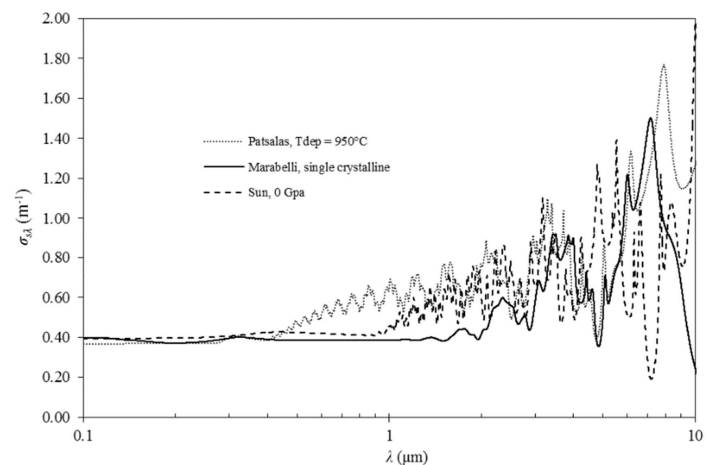

(b)

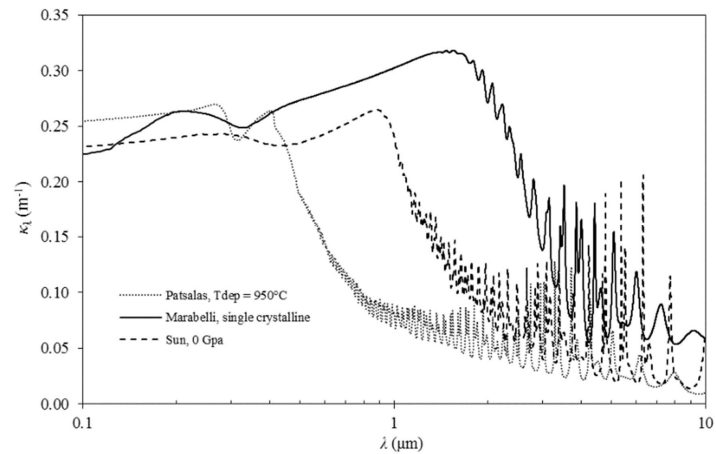

Figure 9. Spectral scattering (a) and absorption (b) coefficients. $\sigma_{\mathrm{s} \lambda}$ and $\kappa_{\lambda}$ calculated from the Mie theory and Eqs. 11, using the three data sets by Marabelli \& Wachter, ${ }^{8}$ Patsalas et al., ${ }^{9}$ and Sun et al.$^{10}$ for the spectral complex refractive index, $m_{\lambda}$. 
(a)

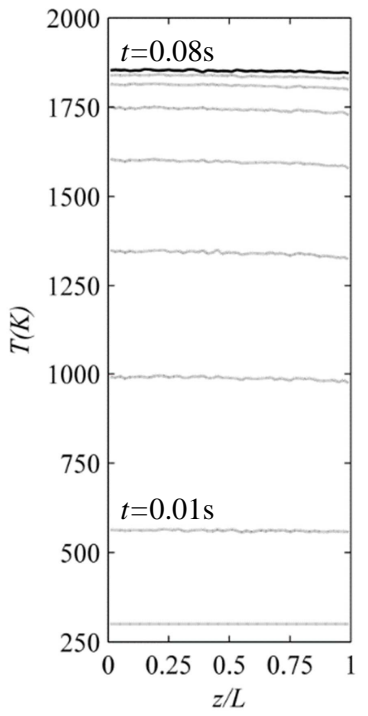

(b)

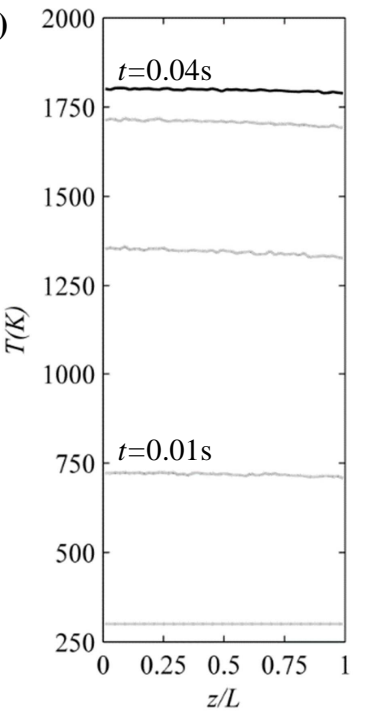

(c)

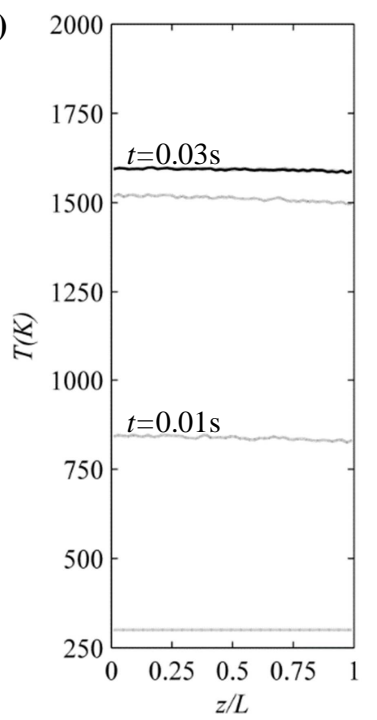

Figure 10. Evolution of the temperature profile in the particle suspension for different underlying data for the complex refractive index. (a) Patsalas et al.,$^{9}$ (b) Sun et al.,$^{10}$ and (c) Marabelli \& Wachter. ${ }^{8}$ The gray curves show the transient temperature profiles at the time interval $\Delta \tau=0.01 \mathrm{~s}$. The black curves are the steady-state temperature profiles.

\section{Summary and conclusions}

Transient partial thermal reduction of a suspension of ceria particles under concentrated solar radiation has been investigated numerically. The particle suspension is modeled as a 1-D infinite slab, externally irradiated at one boundary, with non-participating surroundings. The evolution of the temperature and non-stoichiometry distributions in the particle suspension is studied, varying the particle volume fraction and particle diameter, as well as the unknown kinetic rate constant and the mixing regime of the particles, and using three different data sets for the complex refractive index of ceria.

Increasing the particle volume fraction from $10^{-6}$ to $10^{-3}$ increases the heat capacity of the particle suspension and its optical thickness, leading to lower heating rates, higher peak temperatures, and less uniform temperature and non-stoichiometry distributions. Steady-state temperatures are reached faster than steady-state non-stoichiometries, indicating that the reaction rate is limited by chemical kinetics, not by heat transfer. Both, the final (equilibrium) non-stoichiometry and the reaction rate are sensitive to temperature changes. For example, a change in the volume fraction from $10^{-6}$ to $10^{-4}$ leads to a change in the peak steady-state particle temperature of $230 \mathrm{~K}$ (from 1860 to $2090 \mathrm{~K}$ ). This temperature change nearly doubles the peak equilibrium non-stoichiometry (from 0.095 to 0.18 ), and reduces the time to reach equilibrium steady-state by a factor of 9 (from $3.6 \mathrm{~s}$ to $0.4 \mathrm{~s}$ ). Decreasing particle diameter leads to increasing optical thickness of the particle suspension, resulting in increasing average steady-state particle temperature. A decrease in the particle diameter from 20 to $1 \mu \mathrm{m}$ leads to an increase in the average steady-state temperature from 1703 to $2222 \mathrm{~K}$, resulting in an increase in the average non-stoichiometry from $\sim 0$ to 0.27 . The material morphology influences the complex refractive index of the ceria particles and hence the scattering and absorption coefficients of the particle suspension, which in turn can significantly influence the temperature of the particle suspension.

The results obtained suggest that, when designing a solar reactor to conduct the thermal reduction of ceria, a uniform temperature distribution in the particle suspension should be targeted, in order to reach uniform reaction extents and hence maximum utilization of the reactive material at minimum residence times. Uniform temperature distribution in the particle suspension is achieved when the particles are well mixed in the system, e.g. through a well-designed gas flow pattern.

\section{Acknowledgments}

We thank Dr. Daniel Keene for discussions of the kinetics of non-stoichiometric reduction of ceria. 


\section{References}

${ }^{1}$ Romero, M., and Steinfeld, A., "Concentrating solar thermal power and thermochemical fuels," Energy \& Environmental Science, Vol. 5, Oct. 2012, pp. 9234-9245.

${ }^{2}$ Chueh, W. C., and Haile, S. M., "A thermochemical study of ceria: exploiting an old material for new modes of energy conversion and $\mathrm{CO}_{2}$ mitigation," Philosophical Transactions of the Royal Society A: Mathematical, Physical, and Engineering Sciences, Vol. 368, Jul. 2010, pp. 3269-3294.

${ }^{3}$ Chueh, W. C., and Haile, S. M., "Ceria as a thermochemical reaction medium for selectively generating syngas or methane from $\mathrm{H}_{2} \mathrm{O}$ and $\mathrm{CO}_{2}$," ChemSusChem, Vol. 2, Jan. 2009, pp. 735-739.

${ }^{4}$ Keene, D., Davidson, J. H., and Lipiński, W., "A model of transient heat and mass transfer in a heterogeneous medium of ceria undergoing nonstoichiometric reduction,” Journal of Heat Transfer, Vol. 135, 2013, pp. 052701.

${ }^{5}$ Modest, M. F., Radiative Heat Transfer, $2^{\text {nd }}$ ed., Elsevier Science (USA), 2003.

${ }^{6}$ Bohren, C. F., and Huffman, D. R., Absorption and Scattering of Light by Small Particles, John Wiley \& Sons, New York, 1998.

${ }^{7}$ Panlener, R., Blumenthal, R., and Garnier, J., “A thermodynamic study of nonstoichiometric cerium dioxide," Journal of Physics and Chemistry of Solids, Vol. 3611, 1975, pp. 1213-1222.

${ }^{8}$ Marabelli, F., and Wachter, P., "Covalent insulator $\mathrm{CeO}_{2}$ : optical reflectivity measurements," Physical Review. B, Condensed Matter, Vol. 36, No. 2, 1987, pp. 1238-1243.

${ }^{9}$ Patsalas, P., Logothetidis, S., and Metaxa, C., "Optical performance of nanocrystalline transparent ceria films," Applied Physics Letters, Vol. 81, No. 3, 2002, pp. 466-468.

${ }^{10}$ Sun, L.-L., Cheng, Y., and Ji, G.-F., "Elastic and optical properties of $\mathrm{CeO}_{2}$ via first-principles calculations," Journal of Atomic and Molecular Sciences, Vol. 1, 2010, pp. 143-151.

${ }^{11}$ Binnewies, M., and Milke, E., Thermochemical Data of Elements and Compounds, Weinheim: Wiley-VCH Verlag GmbH, 2002.

${ }^{12}$ Siegel, R., and Howell, J., Thermal Radiation Heat Transfer, $4^{\text {th }}$ ed., Taylor \& Francis, New York, 2002. 